\title{
Integrated analysis of expression profiling data identifies three genes in correlation with poor prognosis of triple-negative breast cancer
}

\author{
CHENG ZHANG, YONG HAN, HAO HUANG, LI MIN, LIKE QU and CHENGCHAO SHOU \\ Key Laboratory of Carcinogenesis and Translational Research (Ministry of Education), Department of Biochemistry \\ and Molecular Biology, Peking University Cancer Hospital and Institute, Beijing 100142, P.R. China
}

Received January 4, 2014; Accepted February 27, 2014

DOI: $10.3892 /$ ijo.2014.2352

\begin{abstract}
Triple-negative breast cancer (TNBC) shows more aggressive clinical behavior and poorer outcome than non-triple-negative breast cancer (NTNBC), and cannot be treated either via endocrine therapy or by Trastuzumab. For TNBC, chemotherapy is currently the mainstay of systemic medical treatment, the lack of more efficient options of treatment has been a problem in breast cancer prevention. In this study, we aimed to find genes related to prognosis in TNBC by bioinformatic analysis and to provide therapeutic candidates for TNBC treatment. We compared the differences in gene expression levels between cancer patients and healthy individuals across five breast cancer microarray databases to generate a gene cohort specifically upregulated in the NTNBC subtype, whose expression levels are $\geq 2$-fold higher in TNBC compared to NTNBC and healthy individuals. Another two databases with clinical information were applied for following Kaplan-Meier analysis, and high expression of BIRC5, CENPA and FAM64A in this cohort were found to be related to poor survival (OS, DMFS, DFS and RFS). This correlation was also seen in patients at early stages and grades. On the other hand, the outcome of patients with synchronous upregulation of these three genes was the worst, while those with synchronous low gene level was the best. In conclusion, BIRC5, CENPA and FAM64A are specifically upregulated in TNBC, and the high expression of these three genes is associated with poor breast cancer prognosis, suggesting their clinical implication as therapeutic targets in TNBC.
\end{abstract}

Correspondence to: Professor Chengchao Shou, Key Laboratory of Carcinogenesis and Translational Research (Ministry of Education), Peking University Cancer Hospital and Institute, 52 Fucheng Road, Beijing 100142, P.R. China

E-mail: scc@bjcancer.org

Key words: triple-negative breast cancer, expression profiling data, prognosis correlated genes

\section{Introduction}

Breast malignancy is the most prevalent type of cancer and the leading cause of cancer death in females around the world (1). According to the presence of ER, PR and HER2, breast cancer can be classified into two molecular subtypes: the triplenegative subtype (all three of ER, PR, HER2 are absent, aka TNBC) and non-triple-negative subtype (at least one receptor remains, aka NTNBC) (2,3).

Traditional anticancer methods, surgery, radiotherapy and chemotherapy are generally used for breast cancer, but they are not so efficient within specific subtypes. For hormone receptorpositive breast cancer, endocrine therapy is a better choice in patients with chronic disease progression. In recent years, with the development of targeted therapies, antibody-based drugs aiming at cancer-specific molecules has been widely applied and turned out to be effective, such as Trastuzumab (Herceptin) against ERBB2 in HER2 ${ }^{+}$subtypes (4). However, no clear consensus has been reached for the treatment of TNBC $(5,6)$. Plenty of clinical reports showed that TN is the subtype of higher malignancy and has a poorer expectation of prognosis and overall survival in breast cancer $(2,7,8)$. Due to the absence of ER, PR and HER2, endocrine treatment and Trastuzumab were invalid for TNBC patients. Current options such as chemotherapy (by platinum compounds or doxorubicin), EGFR antagonists, PARP inhibitors and antiangiogenesis therapy do not act so well against TNBC, and combinations of these agents require further investigation $(5,9)$. As a result, searching for new therapeutic targets for TNBC is of great clinical value.

To investigate genes correlated with the poor prognosis of TNBC and searching for potential TNBC-specific therapeutic targets, we analyzed 5 breast cancer expressing profiling datasets from TCGA and GEO databases (10) and generated a primary set whose expression level was much higher in TNBC patients than in normal controls. To ensure the specificity of these genes in TNBC, corresponding NTNBC expression data were then compared by independent sample T-test. The expression level of 45 genes were found to be statistically higher in TNBC than in NTNBC. Kaplan-Meier analysis for the 45-gene TNBC specific cohort was performed with further two clinical datasets, and within these cohort genes, 
BIRC5, CENPA and FAM64A were found significantly associated with poor breast cancer survival. This correlation was also seen in patients of early cancer stages and grades, and synchronous upregulation of three genes is correlated with the worst prognosis. Analysis of pathway revealed that $3 \mathrm{hub}$ genes were largely co-expressed with many other molecules related to survival, and potential interactions and the related pathways are also discussed. These findings suggested that the high expressions of BIRC5, CENPA and FAM64A are involved with the poor clinical outcome of TNBC, and they are potential prognostic markers and therapeutic targets of triple-negative breast cancer.

\section{Materials and methods}

Ethics statement. The databases searched in our study are available online. We were free to use TCGA breast cancer databases from The Cancer Genome Atlas (https://tcga-data. nci.nih.gov) by meeting its freedom-to-publish criteria. All the Gene Expression Omnibus datasets are also accessible to public from the GEO website (www.ncbi.nlm.nih.gov/geo) and the ROCK breast cancer functional genomics (http://www. rock.icr.ac.uk), an integrated online breast cancer knowledgebase which provides and updates detailed microarray databases and information from reports on breast cancer (11).

Selection of databases. Five breast cancer microarray databases from published studies were used to identify TNBC specific genes. Normalized gene expression profiling data were downloaded from TCGA and GEO websites, while the clinical information was extracted from corresponding reports, TCGA and ROCK websites. Samples were deposited into TNBC, NTNBC and normal groups according to molecular subtyping and immunohistochemical staining results, as presented in Table I.

The two further online clinical datasets were chosen to analyze the association of TNBC specific genes with breast cancer prognosis. NKI data were extracted from a previous study of van de Vijver et al (12), while 2006CC data from the study of Chin et al (13). The median age of patients in NKI and 2006CC data are 44 and 51, respectively, and each dataset contains patients of different lymph status and molecular subtypes. Except for microarray data, NKI dataset contains overall survival (OS), distant metastasis-free survival (DMFS), disease-free survival (DFS) and Nottingham Grading Score (NGS) information, while 2006CC dataset contains OS, DMFS, relapse-free survival (RFS) and TNM staging information.

Identification of TNBC specific genes. Triple-negative subtype constitutes only a small fraction of all breast cancercases, hence to investigate as many breast cancer patients as possible while avoiding cross-study batch effects, we separately carried out statistical analysis in each of these 5 databases (14). Significant analysis of microarray (SAM) method $(\mathrm{p}<0.05, \mathrm{q}<0.25)$ and independent sample t-test $(\mathrm{p}<0.05)$ were used to compare the expression level of each gene between patients and healthy persons. Since the platform and corresponding gene sums of the five datasets were not in concert, to avoid batch effects between experiments while excluding as many heterogenetic
Table I. Statistics of the five microarray databases that generated the 48 -gene primary set.

\begin{tabular}{lrrrr}
\hline Dataset ID & TNBC & NTNBC & N & Total number \\
\hline GSE1992 & 25 & 125 & 8 & 158 \\
GSE2607 & 8 & 36 & 6 & 50 \\
GSE3744 & 18 & 22 & 7 & 47 \\
GSE47561 & 73 & 1457 & 40 & 1570 \\
TCGA & 71 & 398 & 61 & 530 \\
Total number & 195 & 2038 & 122 & 2355 \\
\hline
\end{tabular}

genes as possible, statistical analysis was performed for each dataset, and the overlapping part was output. Overlapping genes upregulated $>2$-fold in patients while with statistical significance $(\mathrm{p}<0.05)$ were deposited into the primary set.

To ensure that members of this gene set were specifically overexpressed in TNBC, we compared corresponding TNBC expression data of genes in the primary set with NTNBC in each database via SAM method or independent sample $\mathrm{t}$-test with the judgment criteria of $\mathrm{p}<0.05$. A cohort of genes statistically upregulated in triple-negative breast cancer was generated and then annotated by DAVID (http://david.abcc. ncifcrf.gov/). The expression intensity of microarray data was demonstrated as heatmap by Mev 4.0 software.

Association of TNBC-upregulated genes with patient prognosis. In the five datasets applied to generate a 45 -gene cohort, four GEO datasets contain no clinical follow-ups, while a large fraction of TCGA survival information were censored or still upgrading. For further analysis, we chose NKI and $2006 \mathrm{CC}$ clinical sets to investigate the association between TNBC cohort genes and breast cancer prognosis. The median of TNBC-upregulated cohort gene levels across samples were calculated to define the attribution of each sample. For each gene, patients with an expression level higher than median was classified as 'high', while those with a level lower than median was classified as 'low'. The relationship between the level of each gene and patient survival [including disease-free survival (DFS), distant metastasis-free survival (DMFS), relapse-free survival (RFS) and overall survival (OS)] was described via Kaplan-Meier analysis in both clinical sets. The survival curves were drawn via GraphPad Prism 5, patients of 'high' or 'low' categories were displayed by curves of different colors, while censored value were displayed as small bars. Genes whose expression level statistically correlated with breast cancer survival in both clinical sets were defined as hub genes.

Tumor grade/stage differentiated relationship of hub genes with survival. To investigate the role of hub genes in survival within different tumor periods, we divided the patients into subgroups according to tumor grade and stage. In two clinical sets applied in this study, NKI contains Nottingham grading score (NGS) information, patients in this set were classified as grade 1-3, while 2006CC contains TNM staging information, patients in this set were classified as stage I-II and stage III-IV. Expression level of each hub gene in each sample were 


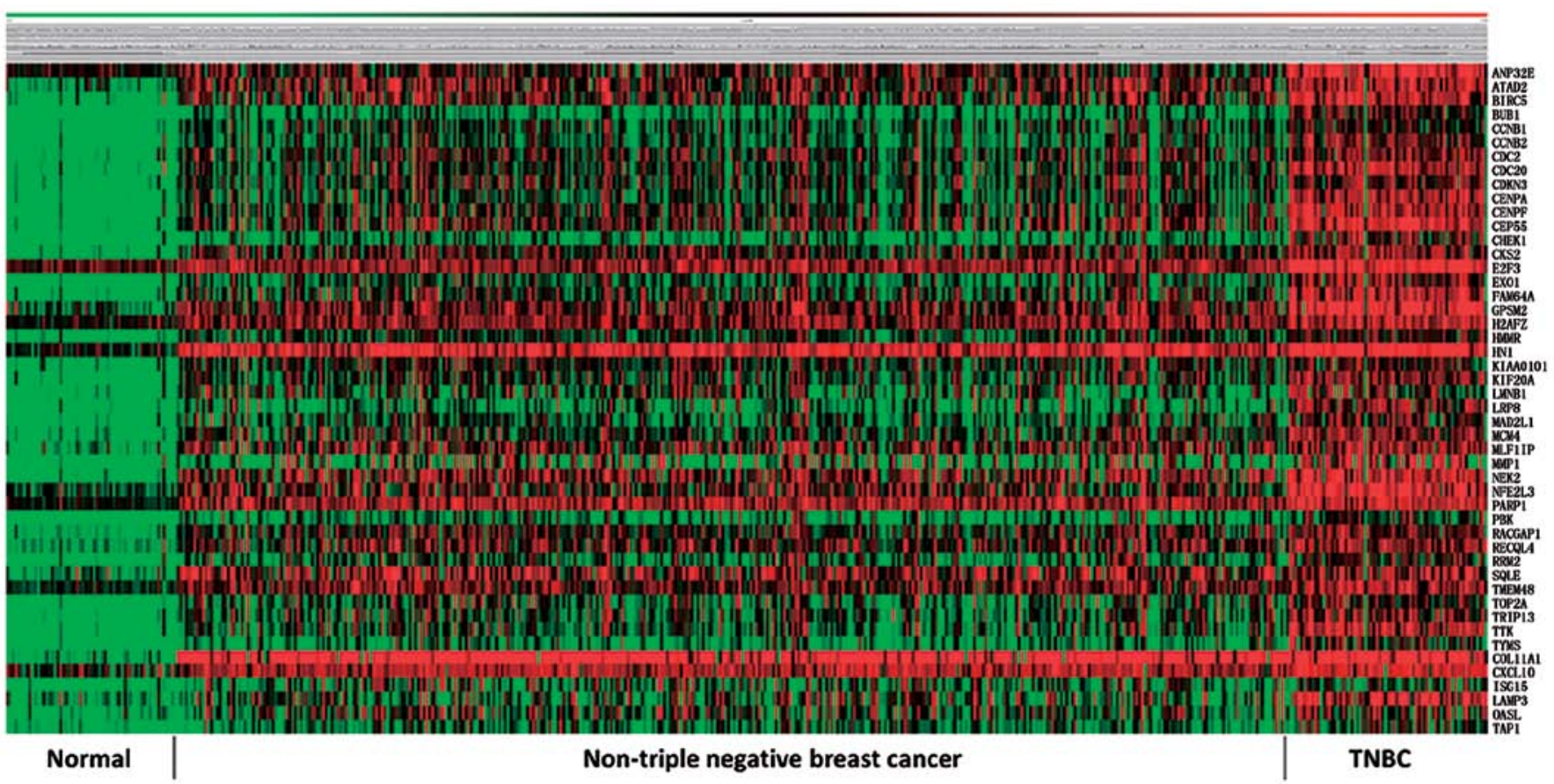

Figure 1. Heatmap for 48 primary set genes in TCGA data. Samples were grouped as normal, NTNBC and TNBC. The expression level of all 48 genes were $\geq 2$-fold higher in TNBC than in normal controls.

defined as 'high' or 'low' by using median as the threshold as mentioned above. Kaplan-Meier analysis of hub gene-related survival was then performed.

Grouped survival analysis of three hub genes. To find out whether identified hub genes were indispensable factors influencing patient survival, we classified samples of both clinical sets into groups according to the level of hub genes. Samples with all hub genes highly expressed were defined as 'triplepositive', samples with all hub genes downregulated were defined as 'triple-negative', while those in the middle were further grouped. Kaplan-Meier analysis was made for each group.

Cox proportional hazards regression analysis of the genes of interest. To weigh the relative influence of the genes on prognosis, Cox proportional hazards regression analysis was performed in two clinical sets using OS and DMFS. Analysis was performed by SPSS 19.0. All the genes of interest were used as covariates, and the 'Forward: LR' method was used.

Pathway analysis of survival associated TNBC genes. In order to further investigate the role of these survival-related genes in breast cancer, we constructed an interaction network using GNCpro online tools (15). Genes and pathways shown in this network were analyzed in NCBI and KEGG, and related reports were downloaded from PubMed.

\section{Results}

Generation of triple-negative breast cancer-specific gene cohort. Definitely molecular subtyped TNBC class covers only $\sim 12-17 \%$ of all breast cancer patients, exclusive of basal-like and BRCA1-related subtypes (5). To expand the sample pool as much as possible, we browsed all available public databases, and those with TNBC samples fewer than 5 were discarded. Only 5 databases were chosen, and the total number of TNBC, NTNBC and normal were 211, 2038 and 122, respectively (16) (Table I). The expression level of triple-negative samples versus normal in GSE3744 (17) and GSE47561 (11) data were compared based on SAM methods (18) with $\mathrm{p}<0.05$ and $\mathrm{q}<0.25$, but for TCGA, GSE2607 (19) and GSE1992 (20) data, independent sample t-test $(\mathrm{p}<0.05)$ was applied instead of SAM because of the existence of numerous databases. A primary set of 48 genes were found to be expressed $\geq 2$-fold higher in TNBC than in healthy controls across the five databases. A heatmap for the 48-gene primary set from TCGA database is shown as Fig. 1.

With a judgment criteria of $\mathrm{p}<0.05$, in all 48 members, a cohort of 45 genes was identified to be statistically upregulated in TNBC compared to NTNBC, 2 genes were of similar levels in TNBC and NTNBC, and 1 gene was downregulated in NTNBC, not in TNBC. These 45 TNBC specific genes were annotated to be related to important cellular mechanisms such as cell cycle regulation, centrosome function, enzymatic canalization and maintenance of chromosome integrity, all of which were potential breach points of carcinogenesis (Table II).

Cohort genes correlated with poor prognosis. The poor clinical outcome and short survival of TNBC might be related to the overexpression of some TNBC specific genes (21). The association between cohort genes and patient survival were analyzed using NKI and 2006CC clinical sets according to procedures mentioned in Materials and methods. We found that among the 45 TNBC-upregulated genes, the overexpression of BIRC5, CENPA and FAM64A showed a significant 
Table II. Fold changes and annotations of 45 TNBC-upregulated genes from TCGA breast cancer data.

\begin{tabular}{|c|c|c|c|}
\hline \multirow[b]{2}{*}{$\begin{array}{l}\text { Gene } \\
\text { symbol }\end{array}$} & \multicolumn{2}{|c|}{ Fold change } & \multirow[b]{2}{*}{ The gene name } \\
\hline & $\begin{array}{l}\text { TNBC } \\
\text { vs. N }\end{array}$ & $\begin{array}{l}\text { TNBC vs. } \\
\text { NTNBC }\end{array}$ & \\
\hline ANP32E & 3.12 & 2.78 & Acidic (leucine-rich) nuclear phosphoprotein 32 family, member E \\
\hline ATAD2 & 4.92 & 1.62 & ATPase family, AAA domain containing 2 \\
\hline BIRC5 & 9.55 & 2.01 & Baculoviral IAP repeat-containing 5 (survivin) \\
\hline BUB1 & 22.53 & 2.94 & BUB1 budding uninhibited by benzimidazoles 1 homolog (yeast) \\
\hline CCNB1 & 5.09 & 1.45 & Cyclin B1 \\
\hline CCNB2 & 8.32 & 2.23 & Cyclin B2 \\
\hline $\mathrm{CDC} 2$ & 6.29 & 1.58 & Cell division cycle $2, \mathrm{G} 1 \rightarrow \mathrm{S}$ and $\mathrm{G} 2 \rightarrow \mathrm{M}$ \\
\hline $\mathrm{CDC} 20$ & 7.83 & 2.69 & CDC20 cell division cycle 20 homolog (S. cerevisiae) \\
\hline CDKN3 & 7.35 & 1.56 & Cyclin-dependent kinase inhibitor 3 \\
\hline CENPA & 12.34 & 2.93 & Centromere protein A \\
\hline CENPF & 15.12 & 2.35 & Centromere protein $\mathrm{F}, 350 / 400 \mathrm{ka}$ (mitosin) \\
\hline CEP55 & 24.52 & 2.74 & Centrosomal protein $55 \mathrm{kDa}$ \\
\hline CHEK1 & 9.64 & 2.88 & CHK1 checkpoint homolog (S. pombe) \\
\hline CKS2 & 7.3 & 1.59 & CDC28 protein kinase regulatory subunit 2 \\
\hline CXCL10 & 3.19 & 1.28 & Chemokine (C-X-C motif) ligand 10 \\
\hline E2F3 & 2.82 & 1.96 & E2F transcription factor 3 \\
\hline EXO1 & 8.54 & 2.38 & Exonuclease 1 \\
\hline FAM64A & 9.97 & 2.59 & Family with sequence similarity 64 , member A \\
\hline GPSM2 & 5.91 & 2.38 & G-protein signalling modulator 2 (AGS3-like, C. elegans) \\
\hline $\mathrm{H} 2 \mathrm{AFZ}$ & 2.36 & 1.41 & $\mathrm{H} 2 \mathrm{~A}$ histone family, member $\mathrm{Z}$ \\
\hline HMMR & 5.23 & 1.49 & Hyaluronan-mediated motility receptor (RHAMM) \\
\hline HN1 & 5.46 & 1.51 & Hematological and neurological expressed 1 \\
\hline KIAA0101 & 4.26 & 1.17 & KIAA0101 \\
\hline KIF20A & 9.7 & 2 & Kinesin family member $20 \mathrm{~A}$ \\
\hline LAMP3 & 8.54 & 4.62 & Lysosomal-associated membrane protein 3 \\
\hline LMNB1 & 6.98 & 1.69 & Lamin B1 \\
\hline LRP8 & 6.87 & 3.43 & Low density lipoprotein receptor-related protein 8 , apolipoprotein $\mathrm{E}$ receptor \\
\hline MAD2L1 & 5.15 & 1.76 & MAD2 mitotic arrest deficient-like 1 (yeast) \\
\hline MCM4 & 5.76 & 1.94 & MCM4 minichromosome maintenance deficient 4 (S. cerevisiae $)$ \\
\hline MLF1IP & 5.59 & 1.44 & MLF1 interacting protein \\
\hline MMP1 & 28.61 & 3.46 & Matrix metallopeptidase 1 (interstitial collagenase) \\
\hline NEK2 & 17 & 1.74 & NIMA (never in mitosis gene a)-related kinase 2 \\
\hline NFE2L3 & 5.26 & 2.99 & Nuclear factor (erythroid-derived 2)-like 3 \\
\hline OASL & 4.15 & 1.29 & 2'-5'-oligoadenylate synthetase-like \\
\hline PARP1 & 2.98 & 1.28 & Poly(ADP-ribose) polymerase family, member 1 \\
\hline $\mathrm{PBK}$ & 11.92 & 1.88 & PDZ binding kinase \\
\hline RACGAP1 & 3.88 & 1.39 & Rac GTPase activating protein 1 \\
\hline RECQL4 & 4.37 & 1.48 & RecQ protein-like 4 \\
\hline RRM2 & 9.81 & 1.81 & Ribonucleotide reductase M2 polypeptide \\
\hline TAP1 & 2.87 & 1.44 & Transporter 1, ATP-binding cassette, sub-family B (MDR/TAP) \\
\hline TMEM48 & 2.83 & 1.46 & Transmembrane protein 48 \\
\hline TOP2A & 8.8 & 1.58 & Topoisomerase (DNA) II $\alpha 170 \mathrm{kDa}$ \\
\hline TRIP13 & 7.06 & 2.08 & Thyroid hormone receptor interactor 13 \\
\hline TTK & 13.81 & 3.27 & TTK protein kinase \\
\hline TYMS & 7.71 & 2.29 & Thymidylate synthetase \\
\hline
\end{tabular}

The expression level of all 45 genes were statistically higher in TNBC than in NTNBC $(\mathrm{p}<0.05)$. 

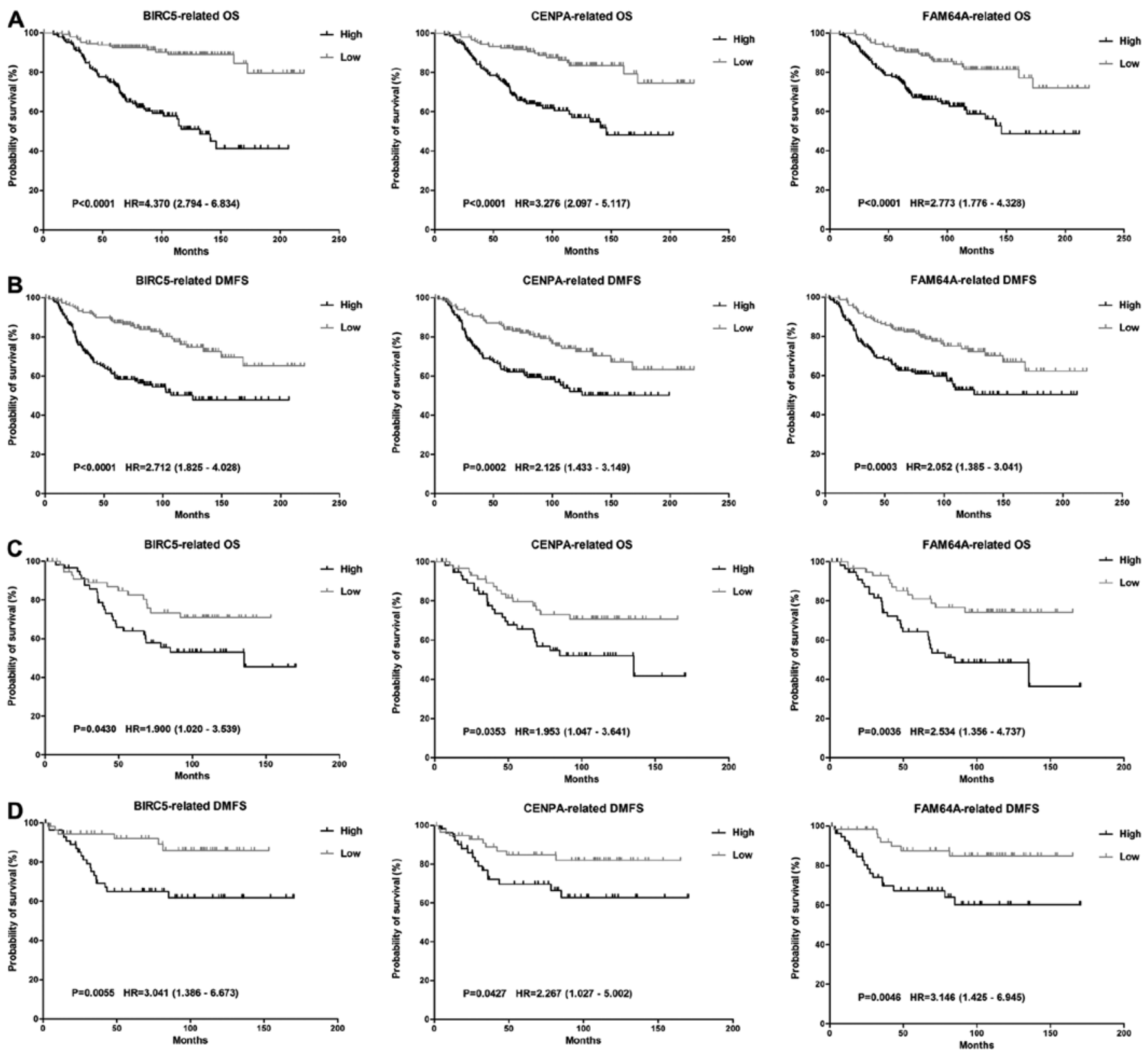

Figure 2. Kaplan-Meier analysis of the hub genes BIRC5, CENPA and FAM64A from two clinical sets. (A) Hub gene related OS in NKI. (B) Hub gene related DMFS in NKI. (C) Hub gene related OS in 2006CC. (D) Hub gene related DMFS in 2006CC.

correlation with poor OS and DMFS $(\mathrm{p}<0.05)$ despite the molecular subtypes of breast cancer (Fig. 2). High expression of these genes were also related with poor DFS and RFS. Therefore, these three genes were classified as hub genes.

Apart from BIRC5, CENPA and FAM64A, some other genes in the cohort were found to be co-expressed with these three hub members and were potentially involved in breast cancer survival as well. In these candidates, the level of BUB1 and CCNB2 genes were also negatively associated with clinical survival, though their relationship with OS in 2006CC data were not statistically supported by log-rank test as with DFS, DMFS or RFS.

Three hub genes are related to survival in early tumor stage/ grade. According to available clinical information, patients in 2006CC were divided as stage I-II and stage III-IV, while patients in NKI clinical set were divided as grade 1-3. Kaplan-Meier analysis showed that patients of stage III-IV have poorer survival than stage I-II, while the rank of survival with NGS was grade $3>2>1$, these results agreed with clinical observations. Furthermore, high level of BIRC5, CENPA and FAM64A were correlated with poor survival in stage I-II patients (Fig. 3). Since the number of advanced tumor samples was too few to perform convincible statistic analysis, we are not sure whether this relationship exists in stage III-IV patients. Similar correlation was also found in patients classified as grade 1-3 by Nottingham grading system.

Synchronous hub gene upregulation is correlated with the worst prognosis. To find out whether these three hub genes were indispensable factors influencing patient survival, we defined patients with all hub genes highly expressed as 'triple-positive' 

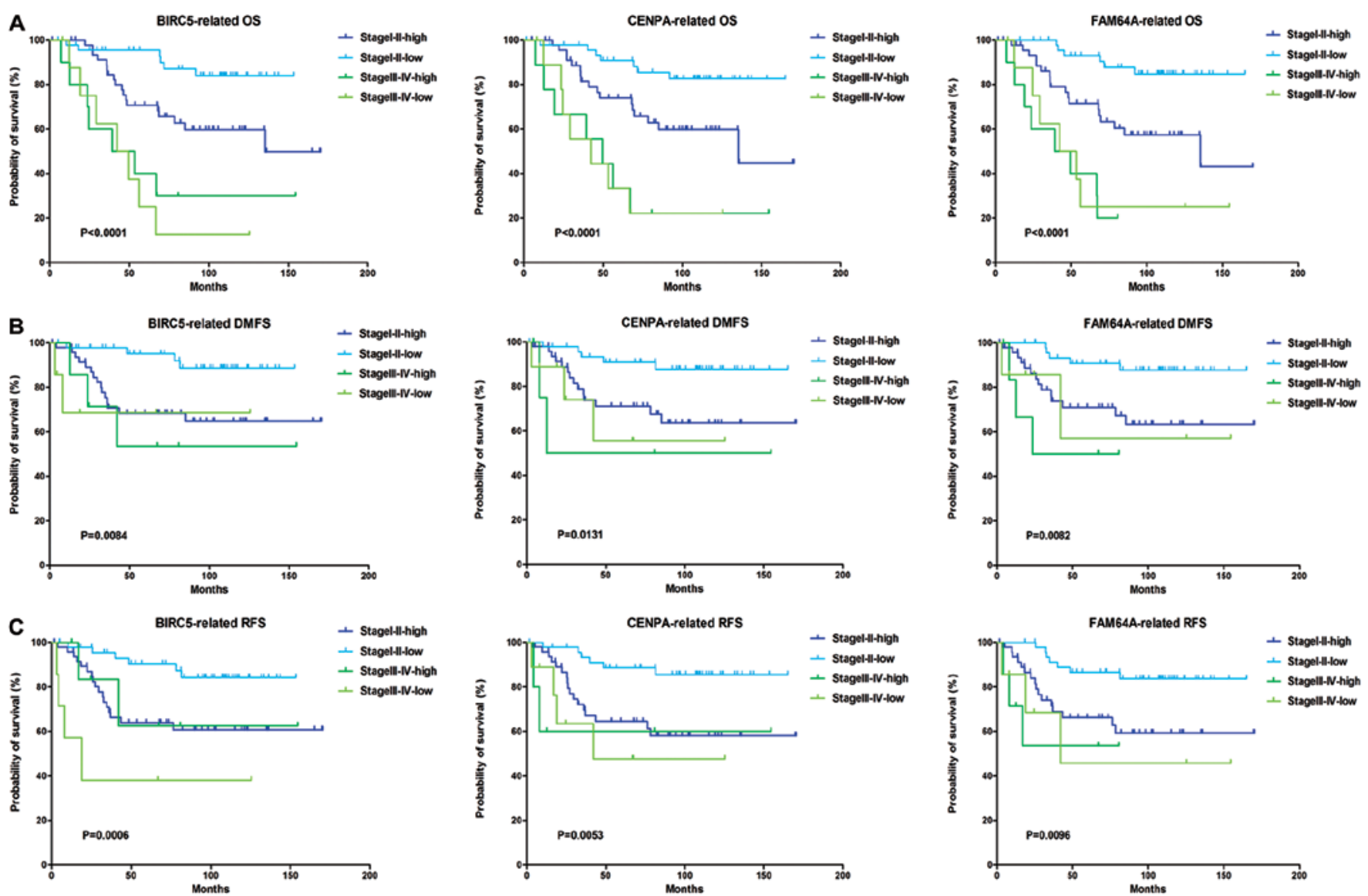

Figure 3. Tumor stage related survival analysis of the hub genes. Samples in 2006CC clinical set were grouped as stage II-III or stage III-IV, hub gene level in each sample was defined as 'high' or 'low'. (A-C) Analysis of OS, DMFS and RFS, respectively.
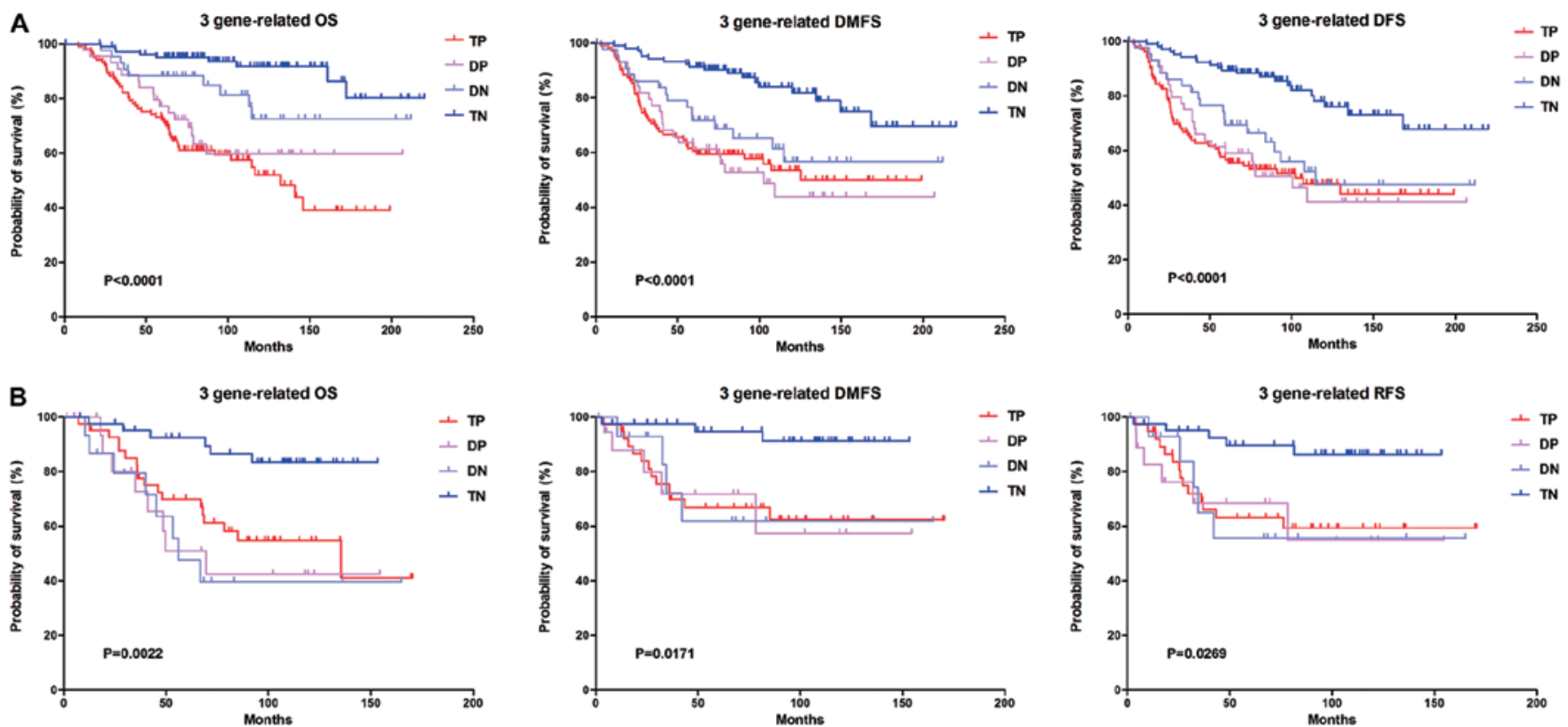

Figure 4. Grouped Kaplan-Meier analysis of the hub genes. TP, triple-positive. DP, double-positive. DN, double-negative. TN, triple-negative. (A) Relationship between grouped hub gene expression and OS, DMFS, DFS in NKI set. (B) Relationship between grouped hub gene expression and OS, DMFS, RFS in $2006 \mathrm{CC}$ set.

(TP), two genes highly expressed as 'double-positive' (DP), 3 genes of low level as 'triple-negative' (TN). Relationships one gene highly expressed as 'double-negative' (DN), and all of these 4 categories with survival were described by Kaplan- 


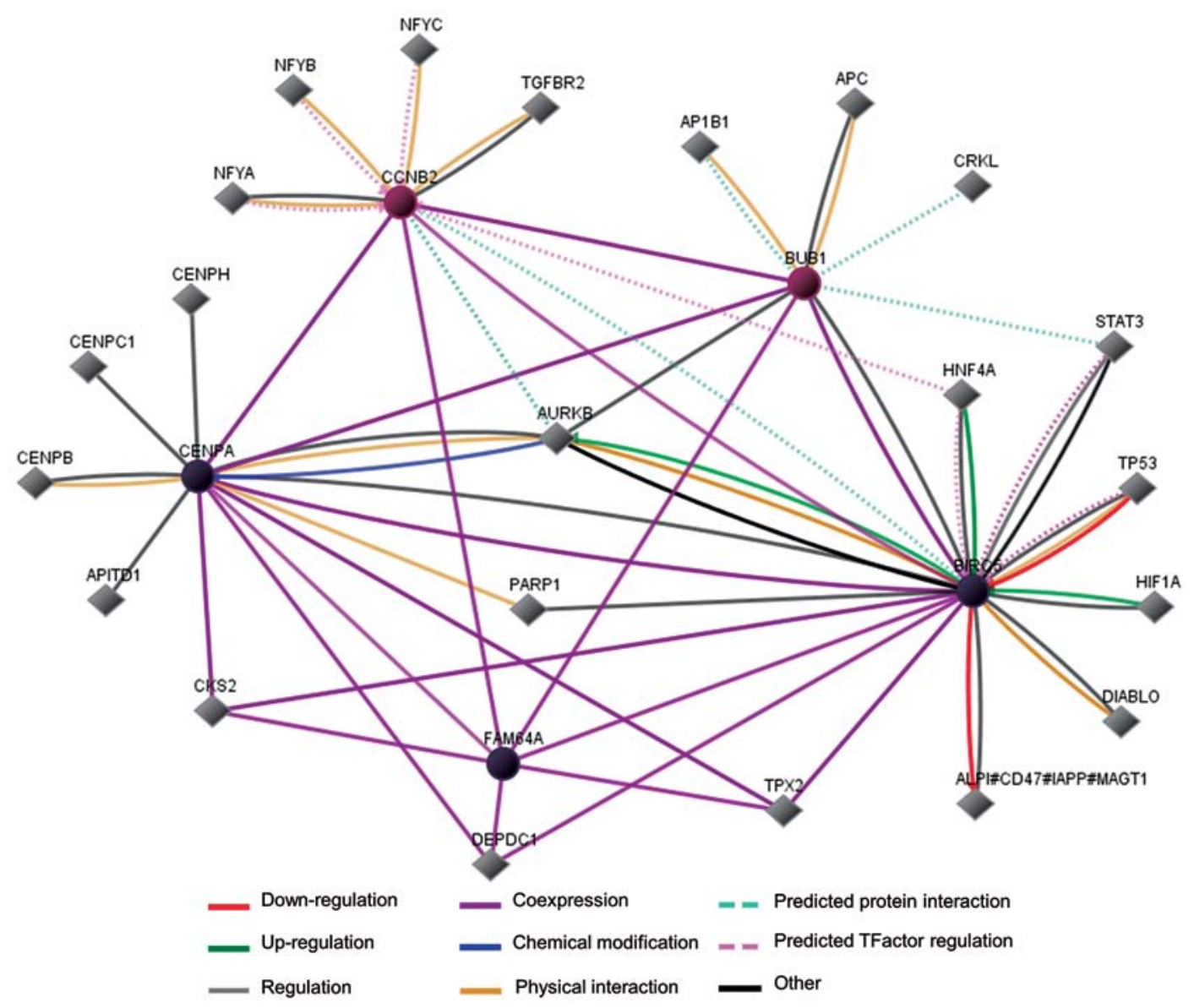

Figure 5. The interaction network of survival related cohort genes. AURKB plays a central role among TNBC-specific genes (BIRC5, CENPA and FAM64A), and its upregulation was also correlated with poor prognosis.

Meier analysis. We found that in NKI data, the TN group had the best OS, DMFS and DFS, while TP was of the worst OS but with similar level of DMFS/DFS as DP and DN groups. In 2006CC data, due to the sample number in each group being limited, survival of TP was similar with DP and NP, but TN still had the best OS, DMFS and RFS (Fig. 4). These results suggested that BIRC5, CENPA and FAM64A are all important molecules influencing survival in breast cancer. In other words, synchronous lower level of three hub genes indicates the best prognosis in breast cancer patients, while synchronous upregulation of three hub genes indicates the worst. Since three hub genes has been previously validated to be upregulated in TNBC subtypes, these findings suggested that the overexpression of BIRC5, CENPA and FAM64A may be the reason for the poor clinical outcome of TNBC, and they are potential therapeutic targets for triple-negative breast cancer.

Cox analysis indicates BIRC5 is an independent prognostic factor in breast cancer. To evaluate the independence of five TNBC-specific genes in inducing poor prognosis, Cox proportional hazards regression analysis was performed in NKI and 2006CC clinical sets according to OS and DMFS. For NKI data, BIRC5 and CCNB2 were considered to be independent prognostic markers for OS and DMFS, while for 2006CC data, BIRC5 and CENPA were considered as independent prognostic markers for OS and DMFS (data not shown), giving a hint that BIRC5 is the more important of these genes in the generation of TNBC high malignancy.

An interaction network of survival related cohort genes. Pathway analysis indicated that BIRC5, CENPA and FAM64A were co-expressed with BUB1 and CCNB2 (Fig. 5). Among the 5 genes, the function of BIRC5, CENPA, BUB1 and CCNB2 have been well studied and proved to be involved with cancer development, while FAM64A is a relatively new molecule with fewer details from NCBI and a limited number of reports. FAM64A was reported to be highly expressed in cancer cell lines and associated with cancer cell cycle regulation and proliferation. In addition, the aurora kinase B gene (aka AURKB, whose protein product regulates chromosome segregation as a serine/threonine kinase) on the map has been confirmed to link BIRC5 and CENPA mechanistically, indicating that AURKB plays an important role among our TNBC-specific genes (Fig. 5).

\section{Discussion}

Although endocrine treatment and targeted drugs against breast cancer have achieved remarkable therapeutic outcomes, the non-triple-negative subtype remains more aggressive and difficult to treat $(22,23)$. The most distinctive difference between TNBC and NTNBC is the status of ER, HER2 and PR (2), which gives the impression that TNBC patient have a 
more similar gene expression signature with healthy people. However, TNBC is indeed one of the most malignant breast subtypes, there might be some unknown molecules and pathways not yet identified. Current therapeutic options of TNBC treatment are chemotherapy and some auxiliary methods (anti-angiogenesis or anti-EGFR) (9), only a small number of previous studies have provided analytical routes and possible gene candidates for targeted treatment of TNBC. In this study, we aimed to find some genes related to prognosis of TNBC by bioinformatic analysis, and provide instructive information for the targeted therapy of TNBC.

After analyzing five online breast cancer microarray datasets, we found a cohort of 45 genes overexpressed in TNBC samples comparing with NTNBC, and in the controls. We checked the relationship between gene level and patient survival. Kaplan-Meier analysis was performed for each gene in the TNBC cohort, and the overexpression of BIRC5, CENPA and FAM64A were found to be associated with poorer OS, DFS, DMFS and RFS. The relationship of the hub genes with survival was also explored according to TNM stage and NGS grade. Since the majority of samples in two clinical sets were located in early or middle stage/grade, as a result, with limited stage III-IV or grade 3 data, it is hard to judge whether the hub genes were related to prognosis in advanced breast cancer. However, this association was clearly seen in patients of stage I-II or grade 1-2, proving that the three hub genes were related to poor prognosis at least in early cancer period. Furthermore, synchronous high expression of these three hub genes indicates the worst clinical outcome while synchronous low level indicates the best, emphasizing their contributions to poor prognosis. On the other hand, two other genes, BUB1 and CCNB2, were also found to be related to DFS, DMFS and RFS. A tendency of correlation with OS was also found, though the log-rank p-value was $>0.05$. Cox model analysis revealed that BIRC5 was more likely to be an independent prognostic factor.

Since the expression of BIRC5, CENPA and FAM64A are significantly upregulated in triple-negative breast cancer, we consider that the overexpression of these genes may be a reason to the high malignancy of TNBC, and they are potential prognostic markers and therapeutic targets in anti-TNBC treatment. BUB1 and CCNB2 may also play some roles influencing TNBC survival.

As the pathway network shows, our interested genes are mainly involved with cell-cycle and mitosis. Among the hub genes, BIRC5 (survivin) has been reported to be related with unfavorable prognostic state $(24,25)$ in cancers of breast $(25)$ and neuroblastoma (26), but its role with survival has not been linked to TNBC. CENPA is known to be a key factor in centrosome functions, but the relationship with breast cancer and prognosis is not well characterized yet. According to its primary functions in mitosis (27), we speculate that CENPA influences survival in cancer by promoting chromosomal segregation, kinetochore association and genomic instability (28). FAM64A is a relatively new molecule, only a few reports exist on its involvement with ovarian cancer cell cycle and proliferation (29), therefore specific mechanisms and pathways on this molecule merit investigation. As for BUB1 and CCNB2, though the relationships with breast cancer has been reported $(30,31)$, their role in TNBC still needs to be further addressed.

According to the interaction map drawn by us, AURKB gene seems to be located at the center of the prognosis-related gene network. This gene encodes a member of the aurora kinase subfamily of serine/threonine kinases, which participate in the regulation of segregation of chromosomes during mitosis and meiosis through association with microtubules, and cancer as well (32). Since AURKB has been reported to be a risk factor in lung and breast malignancy $(33,34)$, it is quite possible that AURKB participates in the regulation of TNBC prognosis. We performed Pearson bivariate correlation analysis for AURKB and the five genes we identified (BIRC5, CENPA, FAM64A, BUB1 and CCNB2). SPSS calculation revealed that the Pearson correlation coefficients between AURKB and each of the five genes were all of statistical significance (data not shown). Exploration of the association between AURKB and patient survival revealed that the level of this gene was negatively correlated with survival similarly to BIRC5, CENPA or FAM64A (data not shown). However, since AURKB remains at a relatively even level among cancer patients, it may play the role of a 'passenger' instead of a 'driver' in TNBC cases.

FAM64A is the substrate of the kinase-interacting stathmin (KIS or UHMK1), a serine/threonine protein kinase that promotes cell cycle progression through G1 by phosphorylation of the cyclin-dependent kinase inhibitor $1 B$ (p27 ${ }^{\text {Kip1 }}$ ) (29). Since AURKB and BUB1 are also serine/ threonine kinases, we doubt whether FAM64A is a substrate of AURKB or BUB1. It has been proven that BIRC5 can upregulate AURKB, while the latter chemically modifies CENPA, so it is possible that BIRC5 exerts the same influence on FAM64A via AURKB or BUB1. We hypothesize that in TNBC patients, except causing poor prognosis, BIRC5 can also promote FAM64A function via AURKB or BUB1 mediated phosphorylation, thus bringing further impairment to clinical outcome in TNBC.

\section{Acknowledgements}

We thank Tiantian She, Hongying Duan and Ting Ma for helpful suggestions. This study was supported by the National Natural Science Foundation of China (81071732).

\section{References}

1. Jemal A, Bray F, Center MM, Ferlay J, Ward E and Forman D: Global cancer statistics. CA Cancer J Clin 61: 69-90, 2011.

2. Dent R, Trudeau M, Pritchard KI, et al: Triple-negative breast cancer: clinical features and patterns of recurrence. Clin Cancer Res 13: 4429-4434, 2007.

3. Rakha EA, El-Sayed ME, Green AR, Lee AH, Robertson JF and Ellis IO: Prognostic markers in triple-negative breast cancer. Cancer 109: 25-32, 2007.

4. Romond EH, Perez EA, Bryant J, et al: Trastuzumab plus adjuvant chemotherapy for operable HER2-positive breast cancer. N Engl J Med 353: 1673-1684, 2005.

5. Foulkes WD, Smith IE and Reis-Filho JS: Triple-negative breast cancer. N Engl J Med 363: 1938-1948, 2010.

6. Mahamodhossen YA, Liu W and Rong-Rong Z: Triple-negative breast cancer: new perspectives for novel therapies. Med Oncol 30: 653, 2013.

7. Cleator S, Heller W and Coombes RC: Triple-negative breast cancer: therapeutic options. Lancet Oncol 8: 235-244, 2007. 
8. Schneider BP, Winer EP, Foulkes WD, et al: Triple-negative breast cancer: risk factors to potential targets. Clin Cancer Res 14: 8010-8018, 2008.

9. Bosch A, Eroles P, Zaragoza R, Viña JR and Lluch A: Triplenegative breast cancer: molecular features, pathogenesis, treatment and current lines of research. Cancer Treat Rev 36: 206-215, 2010.

10. Balko JM, Cook RS, Vaught DB, et al: Profiling of residual breast cancers after neoadjuvant chemotherapy identifies DUSP4 deficiency as a mechanism of drug resistance. Nat Med 18: $1052-1059,2012$.

11. Ur-Rehman S, Gao Q, Mitsopoulos C and Zvelebil M: ROCK: a resource for integrative breast cancer data analysis. Breast Cancer Res Treat 139: 907-921, 2013.

12. van de Vijver MJ, He YD, van't Veer LJ, et al: A gene-expression signature as a predictor of survival in breast cancer. N Engl J Med 347: 1999-2009, 2002.

13. Chin K, DeVries S, Fridlyand J, et al: Genomic and transcriptional aberrations linked to breast cancer pathophysiologies Cancer Cell 10: 529-541, 2006.

14. Butte A: The use and analysis of microarray data. Nat Rev Drug Discov 1: 951-960, 2002.

15. Han Y, Huang H, Xiao Z, et al: Integrated analysis of gene expression profiles associated with response of platinum/paclitaxel-based treatment in epithelial ovarian cancer. PLoS One 7: e52745, 2012.

16. Konstantinopoulos PA, Cannistra SA, Fountzilas H, et al: Integrated analysis of multiple microarray datasets identifies a reproducible survival predictor in ovarian cancer. PLoS One 6 : e18202, 2011.

17. Richardson AL, Wang ZC, De Nicolo A, et al: X chromosomal abnormalities in basal-like human breast cancer. Cancer Cell 9: 121-132, 2006.

18. Brown MP, Grundy WN, Lin D, et al: Knowledge-based analysis of microarray gene expression data by using support vector machines. Proc Natl Acad Sci USA 97: 262-267, 2000.

19. Perreard L, Fan C, Quackenbush JF, et al: Classification and risk stratification of invasive breast carcinomas using a real-time quantitative RT-PCR assay. Breast Cancer Res 8: R23, 2006.

20. Hu Z, Fan C, Oh DS, et al: The molecular portraits of breast tumors are conserved across microarray platforms. BMC Genomics 7: 96, 2006.

21. Gluz O, Liedtke C, Gottschalk N, Pusztai L, Nitz U and Harbeck N: Triple-negative breast cancer - current status and future directions. Ann Oncol 20: 1913-1927, 2009.
22. Rouzier R, Perou CM, Symmans WF, et al: Breast cancer molecular subtypes respond differently to preoperative chemotherapy. Clin Cancer Res 11: 5678-5685, 2005.

23. Vogel CL, Cobleigh MA, Tripathy D, et al: Efficacy and safety of trastuzumab as a single agent in first-line treatment of HER2-overexpressing metastatic breast cancer. J Clin Oncol 20: 719-726, 2002.

24. Hinnis AR, Luckett JC and Walker RA: Survivin is an independent predictor of short-term survival in poor prognostic breast cancer patients. Br J Cancer 96: 639-645, 2007.

25. Span PN, Tjan-Heijnen VC, Heuvel JJ, de Kok JB, Foekens JA and Sweep FC: Do the survivin (BIRC5) splice variants modulate or add to the prognostic value of total survivin in breast cancer? Clin Chem 52: 1693-1700, 2006.

26. Islam A, Kageyama H, Takada N, et al: High expression of Survivin, mapped to $17 \mathrm{q} 25$, is significantly associated with poor prognostic factors and promotes cell survival in human neuroblastoma. Oncogene 19: 617-623, 2000.

27. Regnier V, Vagnarelli P, Fukagawa T, et al: CENP-A is required for accurate chromosome segregation and sustained kinetochore association of BubR1. Mol Cell Biol 25: 3967-3981, 2005.

28. Amato A, Schillaci T, Lentini L and Di Leonardo A: CENPA overexpression promotes genome instability in $\mathrm{pRb}$-depleted human cells. Mol Cancer 8: 119, 2009.

29. Archangelo LF, Greif PA, Maucuer A, et al: The CATS (FAM64A) protein is a substrate of the Kinase Interacting Stathmin (KIS). Biochim Biophys Acta 1833: 1269-1279, 2013.

30. Takagi K, Miki Y, Shibahara Y, et al: BUB1 immunolocalization in breast carcinoma: its nuclear localization as a potent prognostic factor of the patients. Horm Cancer 4: 92-102, 2013.

31. Li L, Xu DB, Zhao XL and Hao TY: Combination analysis of Bub1 and Mad2 expression in endometrial cancer: act as a prognostic factor in endometrial cancer. Arch Gynecol Obstet 288: 155-165, 2013.

32. Hegyi K and Mehes G: Mitotic failures in cancer: Aurora B kinase and its potential role in the development of aneuploidy. Pathol Oncol Res 18: 761-769, 2012.

33. Takeshita M, Koga T, Takayama K, et al: Aurora-B overexpression is correlated with aneuploidy and poor prognosis in non-small cell lung cancer. Lung Cancer 80: 85-90, 2013.

34. Tchatchou S, Wirtenberger M, Hemminki K, et al: Aurora kinases A and B and familial breast cancer risk. Cancer Lett 247: 266-272, 2007. 